\title{
CO-H-SPACES AND ALMOST LOCALIZATION
}

\author{
CRISTINA COSTOYA AND NORIO IWASE
}

\begin{abstract}
Apart from simply-connected spaces, a non simply-connected co-Hspace is a typical example of a space $X$ with a co-action of $B \pi_{1}(X)$ along $r^{X}: X \rightarrow$ $B \pi_{1}(X)$ the classifying map of the universal covering. If such a space $X$ is actually a co-H-space, then the fibrewise $p$-localization of $r^{X}$ (or the 'almost' $p$-localization of $X$ ) is a fibrewise co-H-space (or an 'almost' co-H-space, resp.) for every prime $p$. In this paper, we show that the converse statement is true, i.e., for a non simplyconnected space $X$ with a co-action of $B \pi_{1}(X)$ along $r^{X}, X$ is a co-H-space if, for every prime $p$, the almost $p$-localization of $X$ is an almost co-H-space.
\end{abstract}

\section{Fundamentals and Results}

We assume that spaces have the homotopy type of CW complexes and are based, and maps and homotopies preserve base points. A space $X$ is a co-H-space if there exists a comultiplication, say $\mu^{X}: X \rightarrow X \vee X$ satisfying $j^{X} \circ \mu^{X} \simeq \Delta^{X}$, where $j^{X}: X \vee X \hookrightarrow X \times X$ is the inclusion and $\Delta^{X}: X \rightarrow X \times X$ is the diagonal. Similar to spaces, we say that a group $G$ is a co- $H$-group if there exists a homomorphism $G \rightarrow G * G$ so that the composition with the first and second projections are the identity of $G$. Thus the fundamental group of a co-H-space is a co- $H$-group and the classifying space of a co-H-group is a co-H-space.

When $X$ is a simply-connected co-H-space, the $p$-localization $X_{(p)}$ is also a co-Hspace for any prime $p$. The immediate problem is whether the converse statement holds. In [3], the first author settles the answer in positive when $X$ is a finite simplyconnected complex. In this paper, we extend the above result to non simply-connected spaces in terms of fibrewise $p$-localization (see [1], [14]) or paraphrasing the second author, in terms of almost $p$-localization (see [10]), rather than a usual $p$-localization since the only nilpotent non simply-connected co-H-space is the circle [8].

From now on, we assume that a space $X$ is the total space of a fibrewise space $r^{X}: X \rightarrow B \pi_{1}(X)$, where $r^{X}$ is the classifying map of $c^{X}: \widetilde{X} \rightarrow X$, the universal covering of $X$.

A co-action of $B \pi_{1}(X)$ along $r^{X}$ is a map $\nu: X \rightarrow B \pi_{1}(X) \vee X$ such that when composed with the first projection $B \pi_{1}(X) \vee X \rightarrow B \pi_{1}(X)$ we obtain $r^{X}$ and composed with the second projection $B \pi_{1}(X) \vee X \rightarrow X$ we obtain the identity $1_{X}$. In other words, it consists of a copairing $\nu: X \rightarrow B \pi_{1}(X) \vee X$ with coaxes $r^{X}$ and $1_{X}$ in the sense of Oda [15]. Since the fundamental group of a space $X$ with a co-action of $B \pi_{1}(X)$ along $r^{X}$ is clearly a co- $H$-group, $\pi_{1}(X)$ is free by [5] or [11, and hence $B \pi_{1}(X)$ has the homotopy type of a bunch of circles, say $B$. Thus, $X$ is a fibrewise space over a bunch of circles $B=B \pi_{1}(X)$. Let $s^{X}: B \rightarrow X$ represent the generators of $\pi_{1}(X)$ associated with circles. We may assume that $r^{X} \circ s^{X} \simeq 1_{B}$ and so $X$ is a fibrewise-pointed space over $B$. 
For such a space admitting a co-action of $B$ along $r^{X}: X \rightarrow B$, a retraction map $\rho: B \vee D(X) \rightarrow X$ is constructed in [11, Theorem 3.3.], where $D(X)$ a simplyconnected finite complex. If we factorize $\rho_{\left.\right|_{D}(X)}: D(X) \rightarrow X$ through $\widetilde{X}$, we deduce that if there exists a co-action of $B$ along $r^{X}$, then $X$ is dominated by $B \vee \widetilde{X}$. The space $B$ is a co-H-space and, if $\widetilde{X}$ is a co-H-space, then $X$ is dominated by a co-Hspace hence a co-H-space itself. On the other hand, recall that co-H-spaces are spaces with Lusternik Schnirelmann category, L-S cat, less than or equal to one. Since the L-S cat of $\widetilde{X}$ can not exceed the L-S cat of $X[6]$ if $X$ is a co-H-space then $\widetilde{X}$ is also a co-H-space. Gathering these results altogether and, considering that the almost localization is natural, we obtain the following result.

Proposition 1.1. Let $r^{X}: X \rightarrow B \pi_{1}(X)$ be a fibrewise space over $B=B \pi_{1}(X)$ with a co-action of $B$ along $r^{X}$. Then, the following two statements hold.

1) The space $X$ is a co-H-space if and only if $\tilde{X}$ is a co-H-space.

2) The almost $p$-localization of $X$ for a prime $p, X_{(p)}^{B}$, is also a fibrewise space over $B$ with a co-action of $B$ along the classifying map of the universal covering.

Following earlier authors, e.g., [7, 9, 10, 11], we paraphrase the fibrewise property (see [12, 13]) for a fibrewise based space $r^{X}: X \rightarrow B \pi_{1}(X)$ as 'almost' property for a space $X$. Recall that $X$ is an 'almost' co- $H$-space if there exists a map $\mu$ : $X \rightarrow X \vee_{B} X$, such that when composed with each projection, the identity of $X$ is obtained, where $X \vee_{B} X$ is the pushout of the folding map $\nabla_{B}: B \vee B \rightarrow B$ and $s^{X} \vee s^{X}: B \vee B \rightarrow X \vee X, s^{X}$ section of the classifying map.

Our main result is the following theorem. The rest of this paper will be devoted to prove it.

Theorem 1.2. Let $r^{X}: X \rightarrow B \pi_{1}(X)$ be a fibrewise space over $B=B \pi_{1}(X)$ with a co-action of $B$ along $r^{X}$. If $X$ is a connected finite complex whose almost $p$-localization is an almost co-H-space for every prime $p$, then $X$ is a co-H-space.

Some notation is required. For a set of primes $P$, the almost $P$-localization of $r^{X}: X \rightarrow B$, in other words a fibrewise $P$-localization, is a map $l_{(P)}^{B}: X \rightarrow X_{(P)}^{B}$ which commutes with projections to $B$. The map $l_{(P)}^{B}$ induces an isomorphism of fundamental groups and acts as a standard $P$-localization on the fibre $\widetilde{X}$, so $\widetilde{X_{(P)}^{B}}=$ $\tilde{X}_{(P)}$.

Most of the proofs in this paper follow by induction on the dimension of the space. Henceforth, it will be useful to work with the almost localization introduced by the second author in [10].

\section{2. 'Almost' version of Zabrodsy Mixing}

To show the main result, we need a fibrewise version of a result of Zabrodsky [16, Proposition 4.3.1]. Let $\Pi$ denote the set of all primes, $P$ and $Q$ disjoint sets with $P \sqcup Q=\Pi$. We first give a lemma.

Lemma 2.1. Let $M=B \vee M_{0}$ be the wedge-sum of $B$, a bouquet of 1-dimensional spheres, and $M_{0}$ a simply-connected finite $C W$-complex. Let $X$ be fibrewise pointed 
space over $B$. Then,

$$
[M, X]_{B}=\operatorname{pullback}\left\{\left[\mathrm{M}, \mathrm{X}_{(\mathrm{P})}^{\mathrm{B}}\right]_{\mathrm{B}} \rightarrow\left[\mathrm{M}, \mathrm{X}_{(0)}^{\mathrm{B}}\right]_{\mathrm{B}} \leftarrow\left[\mathrm{M}, \mathrm{X}_{(\mathrm{Q})}^{\mathrm{B}}\right]_{\mathrm{B}}\right\}
$$

Proof. Since we have the following equivalence between homotopy sets,

$$
\left[M, X_{(P)}^{B}\right]_{B}=\left[B \vee M_{0}, X_{(P)}^{B}\right]_{B}=\left[M_{0}, X_{(P)}^{B}\right]=\left[M_{0}, \widetilde{X_{(P)}^{B}}\right]=\left[M_{0}, \widetilde{X}_{(P)}\right],
$$

it is equivalent to proof that

$$
\left[M_{0}, \widetilde{X}\right]=\operatorname{pullback}\left\{\left[\mathrm{M}_{0}, \widetilde{\mathrm{X}}_{(\mathrm{P})}\right] \rightarrow\left[\mathrm{M}_{0}, \widetilde{\mathrm{X}}_{(0)}\right] \leftarrow\left[\mathrm{M}_{0}, \widetilde{\mathrm{X}}_{(\mathrm{Q})}\right]\right\}
$$

This is clear by the fracture square lemma of Bousfield-Kan [2, 6.3.(ii)], since $M_{0}$ is finite and $\widetilde{X}$ is simply-connected.

Proposition 2.2. Let $M$ satisfy the conditions of the previous lemma and $f: M \rightarrow$ $X$ be a fibrewise pointed rational equivalence of fibrewise pointed spaces over $B=$ $B \pi_{1}(M)=B \pi_{1}(X)$ commuting with co-actions of $B$ along the classifying maps of the universal coverings $r^{M}$ and $r^{X}$ respectively. We then have:

1) There exists a unique fibrewise pointed space $M(P, f)$ over $B$ and fibrewise maps $\psi(P, f): M \rightarrow M(P, f)$ and $\phi(Q, f): M(P, f) \rightarrow X$, such that $\psi(P, f)$ is a fibrewise $P$-equivalence, $\phi(Q, f)$ is a fibrewise $Q$-equivalence and the following diagram is homotopy commutative:

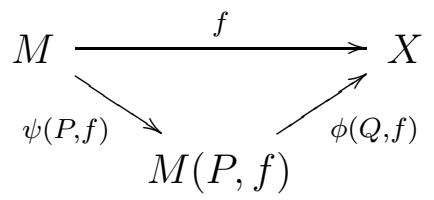

2) The fibrewise pointed space $M(P, f)$ over $B$ and fibrewise maps $\psi(P, f)$ and $\phi(Q, f)$ are natural with respect to $f$, i.e., for a homotopy commutative square:

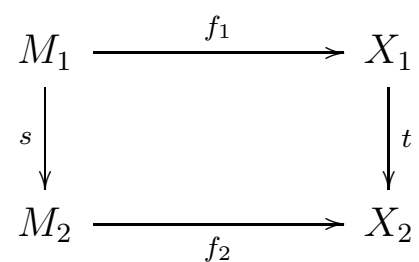

with $f_{1}$ and $f_{2}$ fibrewise rational equivalences, there exists $k\left(P ; f_{1}, f_{2}\right): M\left(P, f_{1}\right) \rightarrow$ $M\left(P, f_{2}\right)$ such that the following diagram is homotopy commutative:

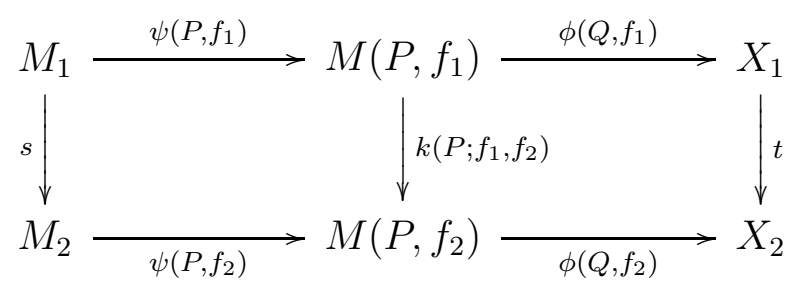

3) If further $M$ and $X$ have the integral homology of a finite space, then so has $M(P, f)$.

Proof. We define $\alpha(P, f)=\ell_{P}^{B} \circ f_{(P)}^{B}=f_{(0)}^{B} \circ \ell_{P}^{B}: M_{(P)}^{B} \rightarrow X_{(0)}^{B}$ and $\beta(Q, f)=\ell_{Q}^{B}$ : $X_{(Q)}^{B} \rightarrow X_{(0)}^{B}$, where by $\ell_{P}^{B}: Y \rightarrow Y_{(0)}^{B}$ we denote the almost rationalization of a 
fibrewise based $P$-local space $r^{Y}: Y \rightarrow B$. With the above maps, we obtain the following commutative diagram:

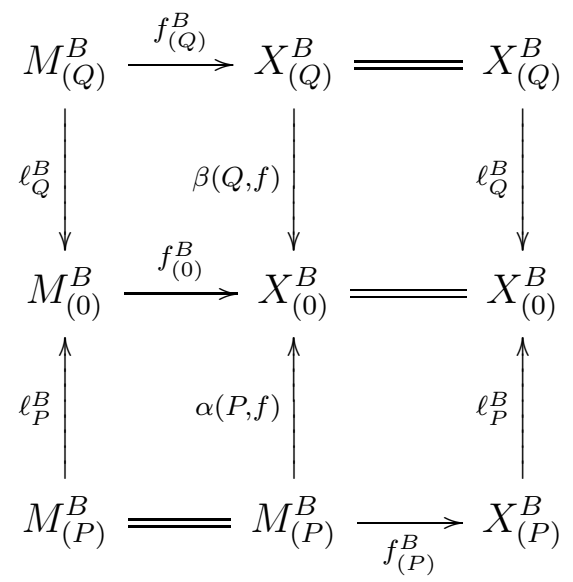

Now, taking the universal covering spaces, we get pullback diagrams by using BousfieldKan's fibre square of localizations [2, p. 127]:

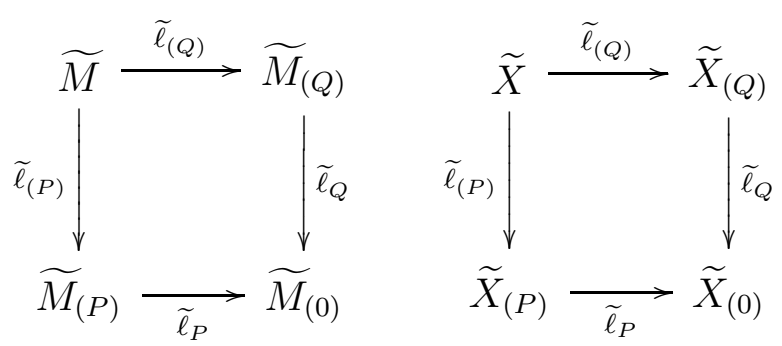

Then, by Theorem 6.3 in [4, we obtain that the following square diagrams are fibrewise homotopy equivalent to fibrewise pullback diagrams:

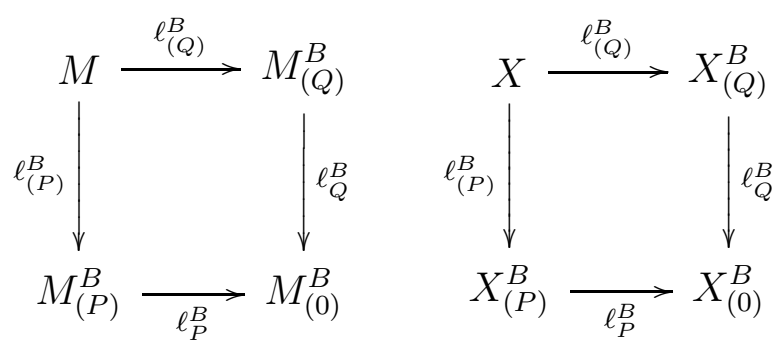

Let the pair of maps $\hat{\alpha}(P, f): M(P, f) \rightarrow X_{(Q)}^{B}$ and $\hat{\beta}(Q, f): M(P, f) \rightarrow M_{(P)}^{B}$ be the fibrewise pullback of $\alpha(P, f)$ and $\beta(Q, f)$ so that $M(P, f)_{(P)}^{B}=M_{(P)}^{B}$ and $M(P, f)_{(Q)}^{B}=X_{(Q)}^{B}$ :

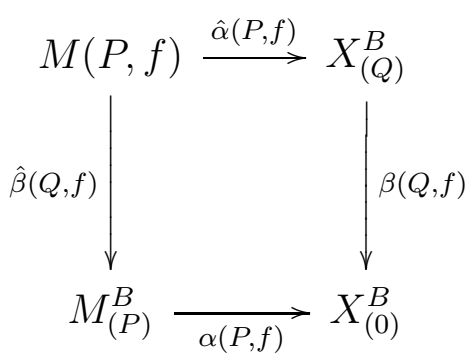

Taking the fibrewise pullback of the vertical arrows in diagram (11), we immediately obtain the existence of maps $\psi(P, f): M \rightarrow M(P, f)$ and $\phi(Q, f): M(P, f) \rightarrow X$ 
which fit within the following commutative diagram:

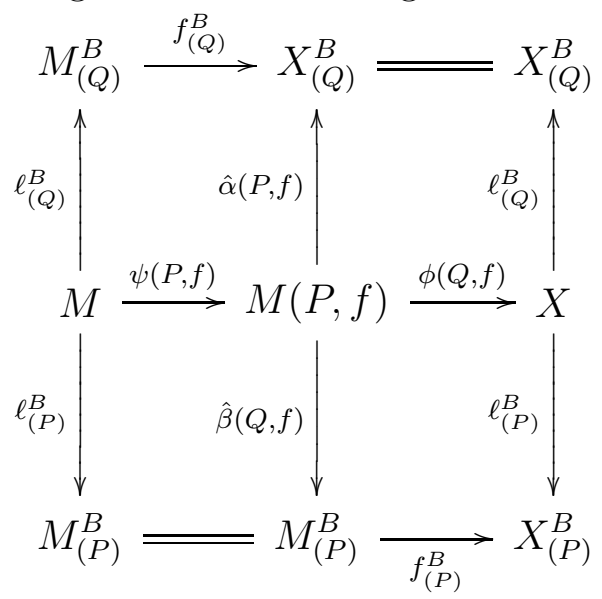

We then have that $f$ and $\phi(Q, f) \circ \psi(P, f)$ have the same almost $P$-localization and $Q$-localization, hence they are equal by Lemma 2.1. Since $f$ is a rational equivalence, $f_{(P)}^{B}$ is a $Q$-equivalence and hence so is $\phi(Q, f)$. Similarly we obtain that $\psi(P, f)$ is a $P$-equivalence. Thus 1 ) in Proposition 2 is proved. The verification of 2) and 3) is straightforward and we leave it to the readers.

Proposition 2.3. For any prime $p$, we have $M(\bar{p}, f)=M\left(\bar{p}, \ell_{(p)}^{B} \circ f\right)$.

Proof. By the unique existence in (1) of Proposition 2.2, the proposition follows from the diagram $M \stackrel{\psi(\bar{p}, f)}{\longrightarrow} M(\bar{p}, f) \stackrel{\ell_{(p)}^{B} \stackrel{\circ}{ } \stackrel{ }{\longrightarrow}(p, f)}{\longrightarrow} X_{(p)}^{B}$, since $\ell_{(p)}^{B}$ is a $p$-equivalence.

We now give a result that will be used in the proof of the main theorem.

Proposition 2.4. Let $f: M \rightarrow X$ be a fibrewise pointed rational equivalence satisfying the conditions of the previous proposition. If $f$ is a fibrewise co-H-map, then $\psi(P, f): M \rightarrow M(P, f)$ is a fibrewise co-H-map.

Proof. We consider the following diagram, where $\rho_{k}^{Y}$ is the projection onto $k$-th factor. Then, the composition of the vertical arrows on the left, and the composition of the vertical arrows on the right, are the identity of $M$ and $X$, respectively. The existence of the dotted arrow is given by (1) in Proposition 2.2.

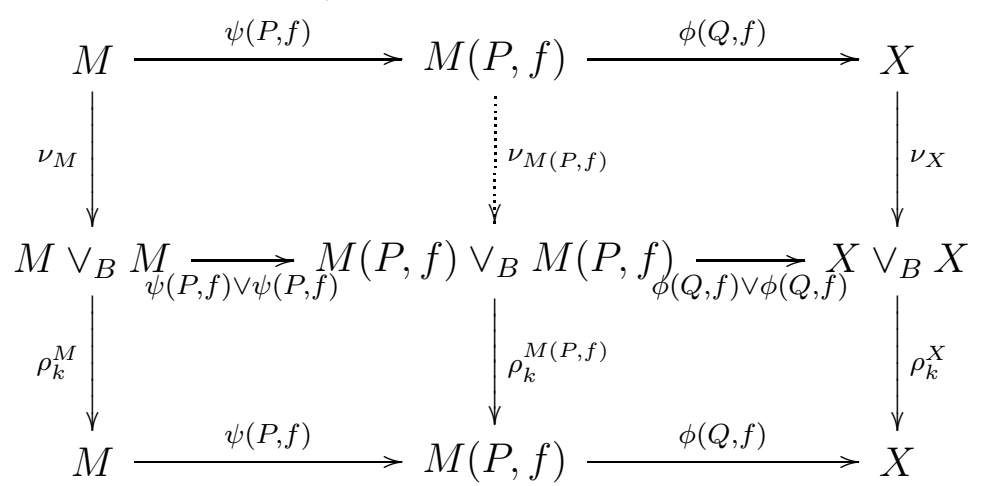

In order to see that $M(P, f)$ is a fibrewise co- $H$-space, it is enough to show that $\rho_{k}^{M(P, f)} \circ \nu_{M(P, f)}$ is a fibrewise homotopy equivalence for $k=1,2$. By the commutativity of the diagram, we can easily see that $\rho_{k}^{M(P, f)} \circ \nu_{M(P, f)}$ induces both a homology 
$\mathbb{Z}_{(P)}$-equivalence and a homology $\mathbb{Z}_{(Q)}$-equivalence on the fibre. Since the fibre of $M(P, f) \rightarrow B$ is simply-connected, $\rho_{k}^{M(P, f)} \circ \nu_{M(P, f)}$ induces a homotopy equivalence on the fibre. Then by [4, Theorem 6.3], we can conclude that $\rho_{k}^{M(P, f)} \circ \nu_{M(P, f)}$ is a fibrewise homotopy equivalence. Thus $M(P, f)$ is an almost co-H-space and the difference between $\rho_{k}^{M(P, f)} \circ \nu_{M(P, f)}$ and the identity of $M(P, f)$ is defined as a fibrewise map $d: M(P, f) \rightarrow M(P, f)$. Again using $P$ and $Q$-localization, we see that $d$ is trivial and hence $\rho_{k}^{M(P, f)} \circ \nu_{M(P, f)}$ is homotopic to the identity. Thus $\psi(P, f): M \rightarrow M(P, f)$ is a fibrewise co-H-map.

From now on, we fix a space $X$ which is a finite complex with a co-action of $B=B \pi_{1}(X)$ along $r^{X}: X \rightarrow B$ the classifying map of the universal covering of $X$. Hence $X$ is a fibrewise pointed space as we mentionned in Section 1. By [10], a co-H-space $X$ has a homology decomposition $\left\{X_{i} ; i \geq 1\right\}$ such that

$$
B=X_{1} \subseteq X_{2} \subseteq \cdots \subseteq X_{n}=X
$$

together with a cofibration sequences $S_{i} \stackrel{h_{i}}{\rightarrow} X_{i} \hookrightarrow X_{i+1}, i \geq 1$, where $S_{i}$ stands for a Moore space of type $\left(H_{i+1}(X), i\right)$.

\section{Fibrewise CO-H-STRUCTURES ON ALmost LOCALiZATions}

By the assumption on a finite complex $X, X_{(p)}^{B}$ is an almost $p$-local co-H-space, which also implies that $X_{(0)}^{B}$ is an almost rational co-H-space. An almost rational co-H-space $X_{(0)}^{B}$ is a wedge-sum of $B$ and finitely many rational spheres of dimension $\geq 2$ [7]. Hence the $k$ '-invariants are all of finite order and we can prove the following lemma.

Lemma 3.1. There is a fibrewise pointed space $M=M(X)$ which is a wedge-sum of a finite number of spheres and, an almost rational equivalence $f=f(X): M \rightarrow X$ which satisfies, for any prime $p$, that there are fibrewise co-H-structures on $M$ such that $\ell_{(p)}^{B} \circ f: M \rightarrow X_{(p)}^{B}$ is a fibrewise co-H-map.

Proof. We construct $M$ and $f$ by induction on the homological dimension of $X$. When $X=X_{1}$, we have nothing to do. So we may assume that $X=X_{i+1}$ and that we have constructed $M_{i}=M\left(X_{i}\right)$ and a fibrewise rational equivalence $f_{i}=f\left(X_{i}\right): M_{i} \rightarrow X_{i}$ satisfying the lemma. Let $d_{i}$ be the order of the $k^{\prime}$-invariant $h_{i}: S_{i} \rightarrow X_{i}$ and $M_{i+1}=M_{i} \vee \Sigma S_{i}^{0}$ where $S_{i}^{0} \subseteq S_{i}$ is the Moore space of type $\left(H_{i+1}(X ; \mathbb{Z}) /\right.$ torsion, $\left.i\right)$. We denote the multiplication by $d_{i}$ by $d_{i}: S_{i} \rightarrow S_{i}$. Let $\nu_{p}: X_{(p)}^{B} \rightarrow X_{(p)}^{B} \vee_{B} X_{(p)}^{B}$ be the given co- $H$-structure on $X_{(p)}^{B}$ and $\nu_{i}^{M}: M_{i} \rightarrow M_{i} \vee_{B} M_{i}$ the co- $H$-structure such that $\ell_{(p)}^{B} \circ f_{i}$ is a fibrewise co- $H$-map. Since $\left(X_{i}\right)_{(p)}^{B}$ gives the homology decomposition of $X_{(p)}^{B}, \nu_{p}$ induces a fibrewise co- $H$-structure $\nu_{p}^{i}$ on $\left(X_{i}\right)_{(p)}^{B}$ by the arguments of [10]. Recall that the $k$-invariants are all of finite order, hence $\left.h_{i} \circ d_{i}\right|_{S_{i}^{0}}=*$ obtaining the 
following commutative diagrams
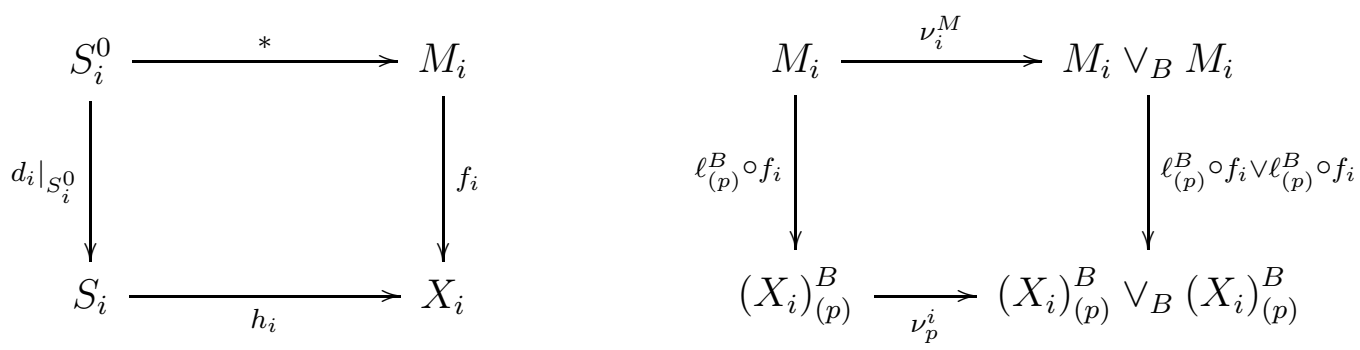

By taking cofibres of the horizontal arrows of the left-hand-side square, we get the following commutative diagram:

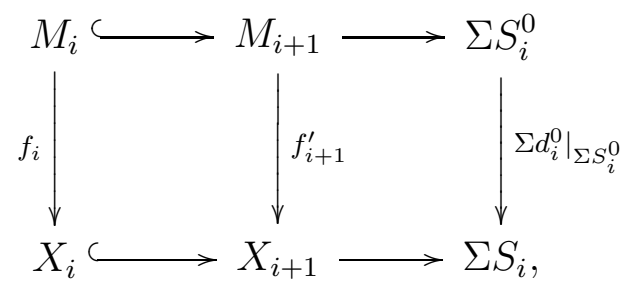

where $M_{i+1}=M_{i} \vee \Sigma S_{i}^{0}$ and $f_{i+1}^{\prime}$ is a rational equivalence, since $f_{i}$ and $\left.\Sigma d_{i}^{0}\right|_{\Sigma S_{i}^{0}}$ are so.

Let $\nu_{i+1}^{\prime M}$ be a fibrewise co- $H$-structure defined by $\left.\nu_{i+1}^{\prime M}\right|_{M_{i}}=\nu_{i}^{M}$ and $\left.\nu_{i+1}^{\prime M}\right|_{\Sigma S_{i}^{0}}$ : $\Sigma S_{i}^{0} \stackrel{\nu_{i}^{S}}{\rightarrow} \Sigma S_{i}^{0} \vee \Sigma S_{i}^{0} \subseteq M_{i+1} \vee_{B} M_{i+1}$, where $\nu_{i}^{S}$ denotes the standard co- $H$-structure of $\Sigma S_{i}^{0}$.

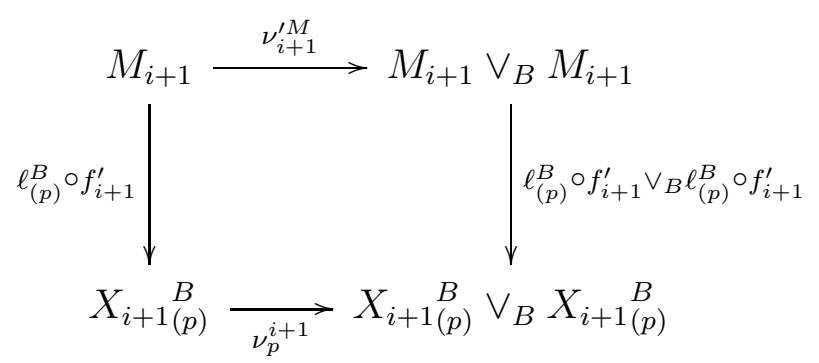

The difference between $\left(\ell_{(p)}^{B} \circ f_{i+1}^{\prime} \vee_{B} \ell_{(p)}^{B} \circ f_{i+1}^{\prime}\right) \circ \nu_{i+1}^{\prime M}$ and $\nu_{p}^{i+1} \circ \ell_{(p)}^{B} \circ f_{i+1}^{\prime}$ is given by a map $D_{i+1}(p): \Sigma S_{i}^{0} \rightarrow \Omega_{B}\left(X_{i(p)}^{B}\right) *_{B} \Omega_{B}\left(X_{i(p)}^{B}\right) \subseteq \Omega_{B}\left(X_{i+1}^{B}(p) *_{B} \Omega_{B}\left(X_{i+1}^{B}(p)\right)\right.$. Since $f_{i+1}^{\prime}$ is a rational equivalence, a multiple $a_{i+1}(p) D_{i+1}(p)$ can be pulled back to $\Omega_{B}\left(M_{i}\right) *_{B} \Omega_{B}\left(M_{i}\right)$. Now, the $k$ '-invariants are of finite order so $X_{i+1}$ and $M_{i+1}$ have the same almost $p$ localization except for primes in $P$, a finite set of primes. Let $a_{i+1}$ be the multiple of $a_{i+1}(p)$ 's for all $p \in P$. Let $f_{i+1}: M_{i+1} \rightarrow X_{i+1}$ be a map defined by $\left.f_{i+1}\right|_{M_{i}}=f_{i}$ and by $\left.f_{i+1}\right|_{\Sigma S_{i}^{0}}=\left.a_{i+1} f_{i+1}^{\prime}\right|_{\Sigma S_{i}^{0}}: \Sigma S_{i}^{0} \rightarrow X_{i+1}$. Then we choose a map $D_{i+1}^{M}(p)$ : $\Sigma S_{i}^{0} \rightarrow \Omega_{B}\left(M_{i}\right) *_{B} \Omega_{B}\left(M_{i}\right)$ as a pull-back of $a_{i+1} D_{i+1}(p)$ onto $\Omega_{B}\left(M_{i}\right) *_{B} \Omega_{B}\left(M_{i}\right)$. The co- $H$-structure defined by $\left.\nu_{i+1}^{M}\right|_{M_{i}}=\nu_{i}^{M}$ and by $\left.\nu_{i+1}^{M}\right|_{\Sigma S_{i}^{0}}=\left.\nu_{i+1}^{\prime M}\right|_{\Sigma S_{i}^{0}}+D_{i+1}^{M}: \Sigma S_{i}^{0} \rightarrow$ $M_{i+1} \vee_{B} M_{i+1}$, together with $\nu_{i+1}^{p}$ verify that $\ell_{(p)}^{B} \circ f_{i+1}$ is a fibrewise co- $H$-map at any prime $p \in P$. For a prime $q \notin P$, we may define comultiplication of $\left(X_{i+1}\right)_{(q)}^{B}$ by that of $\left(M_{i+1}\right)_{(q)}^{B}$, since $\left(X_{i+1}\right)_{(q)}^{B}=\left(M_{i+1}\right)_{(q)}^{B}$. Hence the induction holds and we obtain the lemma. 


\section{Proof of Theorem 1.2}

Let $P_{1}$ and $P_{2}$ be disjoint sets of primes. Then we have a commutative diagram

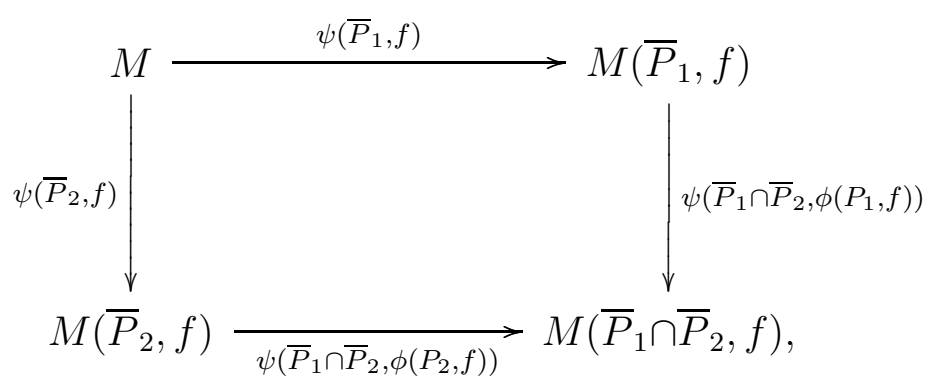

where the vertical arrows are $P_{1}$-equivalences and the horizontal arrows are $P_{2^{-}}$ equivalences.

Proposition 4.1. Assume that $M$ and the spaces $M\left(\bar{P}_{i}, f\right)$ for $i=1,2$ admit fibrewise co-H-structures $\nu, \nu^{P_{i}}$ for $i=1,2$, respectively, such that $\psi\left(\bar{P}_{i}, f\right)$ are fibrewise co-H-maps, for $i=1,2$. Then, $M\left(\bar{P}_{1} \cap \bar{P}_{2}, f\right)$ is a fibrewise co-H-space $\left(P_{1} \cup P_{2}\right)$ equivalent to $X$.

Proof. The commutativity of the diagram (2) gives a map from the pushout $W$ of $M\left(\bar{P}_{1}, f\right)$ and $M\left(\bar{P}_{2}, f\right)$ to $M\left(\bar{P}_{1} \cap \bar{P}_{2}, f\right)$, which induces an isomorphism of homology groups. Thus it follows that $W$ and $M\left(\bar{P}_{1} \cap \bar{P}_{2}, f\right)$ have the same fibre homotopy type. Since a fibrewise pushout of fibrewise co- $H$-structures is a fibrewise co- $H$-space, we obtain that $M\left(\bar{P}_{1} \cap \bar{P}_{2}, f\right)$ is also a fibrewise co- $H$-space such that $\psi\left(\bar{P}_{1} \cap \bar{P}_{2}, f\right)$ : $M \rightarrow M\left(\bar{P}_{1} \cap \bar{P}_{2}, f\right)$ is a fibrewise co- $H$-map.

Now, it suffices to consider $\phi\left(P_{1}, f\right): M\left(\bar{P}_{1}, f\right) \rightarrow X$ and $\phi\left(P_{2}, f\right): M\left(\bar{P}_{2}, f\right) \rightarrow X$, making the diagram commutative, to conclude that the fibrewise pushout is $\left(P_{1} \cup P_{2}\right)$ equivalent to $X$.

Lemma 4.2. Let $f: M \rightarrow X$ be the map constructed in Lemma 3.1, where $M$ is a wedge-sum of a finite number of spheres. Suppose that $X_{\left(P_{1}\right)}^{B}$ and $X_{\left(P_{2}\right)}^{B}$ are fibrewise co-H-spaces with fibrewise co-H-structures $\nu_{1}$ and $\nu_{2}$, respectively, such that $\ell_{\left(P_{1}\right)^{\circ}}^{B} f$ and $\ell_{\left(P_{2}\right)}^{B} \circ f$ are fibrewise co-H-maps with respect to co- $H$-structures on $M, \nu^{\prime}$ and $\nu^{\prime \prime}$ respectively. Then, there exist fibrewise co-H-structures $\nu^{0}$ on $M, \nu^{P_{1}}$ on $X_{\left(P_{1}\right)}^{B}$ and $\nu^{P_{2}}$ on $X_{\left(P_{2}\right)}^{B}$ such that $\ell_{\left(P_{1}\right)}^{B} \circ f$ and $\ell_{\left(P_{2}\right)}^{B}$ of are fibrewise co-H-maps with respect to $\nu^{0}$, $\nu^{P_{1}}$ and $\nu^{P_{2}}$ respectively.

Proof. Firstly remark that, to assume that there exist fibrewise co- $H$-structures on $M$ such that $\ell_{\left(P_{1}\right)}^{B} \circ f: M \rightarrow X_{\left(P_{1}\right)}^{B}$ and $\ell_{\left(P_{2}\right)}^{B} \circ f: M \rightarrow X_{\left(P_{2}\right)}^{B}$ are fibrewise co- $H$-maps, is equivalent by Proposition 2.4 to assume that, there exist fibrewise co- $H$-structures on $M$ such that $\psi\left(\bar{P}_{1}, f\right): M \rightarrow M\left(\bar{P}_{1}, f\right)$ and $\psi\left(\bar{P}_{2}, f\right): M \rightarrow M\left(\bar{P}_{2}, f\right)$ are fibrewise co- $H$-maps. To simplify notation, we will also refer to them as $\nu^{P_{1}}$ on $M\left(\bar{P}_{1}, f\right)$ and $\nu^{P_{2}}$ on $M\left(\bar{P}_{2}, f\right)$.

Now, since $M$ has the homotopy type of a wedge of spheres, there is a standard fibrewise co- $H$-structure $\nu$ on $M$ induced from the unique structure maps on spheres. Let us denote by $d\left(\nu, \nu^{\prime \prime}\right), d\left(\nu, \nu^{\prime \prime}\right): M \rightarrow \Omega_{B}(M) *_{B} \Omega_{B}(M)$ the fibrewise difference of the fibrewise co- $H$-structure maps $\nu, \nu^{\prime}$ and $\nu^{\prime \prime}$. 
The proof will follow by induction on the homological decomposition of $M$. Since the fibrewise differences $d\left(\nu, \nu^{\prime}\right), d\left(\nu, \nu^{\prime \prime}\right)$ and $d\left(\nu^{\prime}, \nu^{\prime \prime}\right)$ are trivial on $M_{1}=M\left(X_{1}\right)=$ $B$, we put $\nu_{1}=\nu, \nu_{1}^{\prime}=\nu^{\prime}, \nu_{1}^{\prime \prime}=\nu^{\prime \prime}, \nu_{1}^{P_{1}}=\nu^{P_{1}}$ and $\nu_{1}^{P_{2}}=\nu^{P_{2}}$.

Secondly, let us assume that there are fibrewise co- $H$-structures $\nu_{i}^{\prime}$ and $\nu_{i}^{\prime \prime}$ on $M$, $\nu_{i}^{P_{1}}$ on $M\left(\bar{P}_{1}, f\right)$ and $\nu_{i}^{P_{2}}$ on $M\left(\bar{P}_{2}, f\right)$ such that $\psi\left(\overline{P_{1}}, f\right)$ and $\psi\left(\overline{P_{2}}, f\right)$ are fibrewise co- $H$-maps. We also assume that $d\left(\nu, \nu_{i}^{\prime}\right)$ and $d\left(\nu, \nu_{i}^{\prime \prime}\right)$ coincide on $M_{i}=M\left(X_{i}\right)$ as induction hypotheses. Since $\psi\left(\overline{P_{1}}, f\right)$ is $\overline{P_{1}}$-equivalence, there are extensions $d^{P_{1}}\left(\nu, \nu_{i}^{\prime}\right)$ and $d^{P_{1}}\left(\nu, \nu_{i}^{\prime \prime}\right)$ of $s d\left(\nu, \nu_{i}^{\prime}\right)$ and $s d\left(\nu, \nu_{i}^{\prime \prime}\right)$ on $M\left(\overline{P_{1}}, f\right)$ for some $s$ such that $\left(s, \overline{P_{1}}\right)=1$. Similarly, we have extensions $d^{P_{2}}\left(\nu, \nu_{i}^{\prime}\right)$ and $d^{P_{2}}\left(\nu, \nu_{i}^{\prime \prime}\right)$ of $t d\left(\nu, \nu_{i}^{\prime}\right)$ and $t d\left(\nu, \nu_{i}^{\prime \prime}\right)$ on $M\left(\overline{P_{2}}, f\right)$ for some $t$ such that $\left(t, \overline{P_{2}}\right)=1$.

Since $P_{1} \cap P_{2}=\emptyset$, we can choose integers $n$ and $m$ such that $n s+m t=1$. Let $\nu_{i+1}^{P_{1}}=\nu_{i}^{P_{1}}-n d^{P_{1}}\left(\nu, \nu_{i}^{\prime}\right)+n d^{P_{1}}\left(\nu, \nu_{i}^{\prime \prime}\right)$ and $\nu_{i+1}^{P_{2}}=\nu_{i}^{P_{2}}+m d^{P_{2}}\left(\nu, \nu_{i}^{\prime}\right)-m d^{P_{2}}\left(\nu, \nu_{i}^{\prime \prime}\right)$, where the sum is taken by $\nu_{i}^{P_{1}}$ and $\nu_{i}^{P_{2}}$ on $M\left(\overline{P_{1}}, f\right)$ and $M\left(\overline{P_{2}}, f\right)$, respectively. Then $\nu_{i+1}^{P_{1}}$ and $\nu_{i+1}^{P_{2}}$ give fibrewise co- $H$-structures on $M\left(\overline{P_{1}}, f\right)$ and $M\left(\overline{P_{2}}, f\right)$.

Similarly, let $\nu_{i+1}^{\prime}=\nu_{i}^{\prime}-n s d\left(\nu, \nu_{i}^{\prime}\right)+n s d\left(\nu, \nu_{i}^{\prime \prime}\right)$ and $\nu_{i+1}^{\prime \prime}=\nu_{i}^{\prime \prime}+m t d\left(\nu, \nu_{i}^{\prime}\right)-$ $m t d\left(\nu, \nu_{i}^{\prime \prime}\right)$, where the sum is taken by $\nu_{i}^{\prime}$ and $\nu_{i}^{\prime \prime}$ on $M$, respectively. By construction, $\psi\left(\overline{P_{1}}, f\right)$ and $\psi\left(\overline{P_{2}}, f\right)$ are fibrewise co- $H$-maps with respect to the fibrewise co- $H$ structures $\nu_{i+1}^{\prime}$ and $\nu_{i+1}^{\prime \prime}$ on $M$, and $\nu_{i+1}^{P_{1}}$ and $\nu_{i+1}^{P_{2}}$, respectively.

Now, using the fact that $n s+m t=1$, and also by classical arguments of connectivity, one can prove that $d\left(\nu, \nu_{i+1}^{\prime}\right)$ coincides with $d\left(\nu, \nu_{i+1}^{\prime \prime}\right)$ over $M_{i+1}$. Thus, by induction on $i$, we obtain the lemma.

By Lemma 4.2, we inductively obtain that $X$ is a fibrewise co- $H$-space, and therefore $\widetilde{X}$ is a co- $H$-space. Hence, by Proposition 1.1, we obtain that $X$ is a co- $H$-space. This completes the proof of Theorem 1.2.

Remark 4.3. Notice that Theorem 1.2 cannot be directly obtained from [3, Theorem 1.1]. Indeed, following along the lines of the previous paragraph, as $X_{(p)}^{B}$ is a fibrewise co-H-space, then $\tilde{X}_{(p)}$ is a co-H-space for every prime $p$. Since we are now in the simply-connected case, one could be tempted to use [3] to conclude that $\tilde{X}$ is a co$H$-space (therefore $X$ is so, by Proposition 1.1). Unfortunately, results in [3] are obtained for finite-type spaces while $\widetilde{X}$ is not.

\section{REFERENCES}

[1] M. Bendersky, A functor which localizes the higher homotopy groups of an arbitrary $C W$ complex, Lecture Notes in Math. 418, Springer Verlag, Berlin (1975), 13-21.

[2] A. K. Bousfield and D. M. Kan, Homotopy limits, completions and localizations, Lecture notes in Mathematics, 304, Springer, Berlin, 1972.

[3] C. Costoya, Co-H-spaces and localization, Isr. Journal of Math. 180 (2010), 69-92.

[4] Dold, A., Partitions of Unity in the Theory of Fibrations, Ann. of Math. 78 (1963), 223-255.

[5] S. Eilenberg and T. Ganea, On the Lusternik-Schnirelmann category of abstract groups, Ann. of Math. 65 (1957), 517-518.

[6] R. H. Fox, On the Lusternik-Schnirelmann category, Ann. of Math. (2) 42, (1941), 333-370.

[7] W. Henn, On almost rational co-H-spaces, Proc. Amer. Math. Soc. 87, (1983), 164-168.

[8] P. Hilton, G. Mislin and J. Roitberg, On co-H-spaces, Comment. Math. Helv. 53 (1978), 1-14.

[9] J. R. Hubbuck and N. Iwase, A p-complete version of the Ganea conjecture on co-H-spaces, "Lusternik-Schnirelmann category and related topics (South Hadley, 2001)", 127-133, Cont. Math., 316, Amer. Math. Soc., Providence, 2002. 
[10] N. Iwase, Co-H-spaces and the Ganea conjecture, Topology 40 (2001), 223-234.

[11] N. Iwase, S. Saito and T. Sumi, Homology of the universal covering space of a co-H-space, Trans. Amer. Math. Soc. 351 (1999), 4837-4846.

[12] I. M. James, Fibrewise Topology, Cambridge University Press, Cambridge 1989.

[13] I. M. James, Introduction to fibrewise Homotopy Theory, Handbook of algebraic topology, 169194, North Holland, Amsterdam, 1995.

[14] J. P. May, Fiberwise localization and completion, Trans. Amer. Math. Soc. 258 (1980), 127-146.

[15] N. Oda, Pairings and copairings in the category of topological spaces, Publ. RIMS Kyoto Univ. 28 (1992), 83-97.

[16] A. Zabrodsky, Hopf spaces, Notas de Matemática (59), North-Holland Publ. Co., Amsterdam, North-Holland Mathematics Studies, 22, 1976.

(C. Costoya) Departamento de Computación, Álxebra, Universidade da Coruña, CamPus de ElviñA, s/n, 15071 - A Coruña, Spain.

E-mail address: cristina.costoya@udc.es

(N. Iwase) Faculty of Mathematics, Kyushu University, Fukuoka 810-8560, Japan

E-mail address: iwase@math.kyushu-u.ac.jp 